\title{
Comparison of proinsulin to C-peptide ratio in children with and without type 1 diabetes and its relation to age
}

\author{
Benjamin R. Ellison ${ }^{1}$, \\ Jeffrey H. Burton, $\mathrm{PhD}^{2}$, \\ Daniel S. Hsia, MD² \\ 'Baylor University, Waco, TX, USA \\ 2Pennington Biomedical Research \\ Center, Baton Rouge, LA, USA
}

Purpose: This study aimed to compare the proinsulin to C-peptide (PI:C) ratio in those with recent-onset type 1 diabetes versus those with no diabetes and to explore the effect of age on PI:C ratio.

Methods: Nineteen participants ( $n=9$ with type 1 diabetes and $n=10$ with no diabetes) between 10 and 19 years of age were enrolled in a single-visit crosssectional study and underwent blood collection after 10 hours fasting to measure proinsulin and C-peptide levels as well as other glycemic parameters.

Results: The median PI:C ratio was significantly different between type 1 diabetes and nondiabetes groups $(6.24 \%$ vs. $1.46 \%, P<0.01)$. A significant negative correlation was seen between $\mathrm{PI}: \mathrm{C}$ ratio and patient age after adjustment for duration of diabetes $\left(r^{2}=0.61, P=0.02\right)$ in the type 1 diabetes group.

Conclusion: Even in this narrow age window, a higher degree of $\beta$-cell dysfunction indicated by a higher PI:C ratio was seen in younger children.

Keywords: Proinsulin, C-peptide, Type 1 diabetes mellitus, Biomarkers

\section{Introduction}

The proinsulin to $\mathrm{C}$-peptide (PI:C) ratio may be an indicator of chronic $\beta$-cell stimulation in conditions with $\beta$-cell loss as well as of secretion of immature insulin granules. ${ }^{1)}$ Increased secretion of proinsulin relative to fully processed insulin also may be a marker of $\beta$-cell endoplasmic reticulum dysfunction. ${ }^{2}$ Moreover, elevated PI:C ratio has been observed in patients with both type 1 and type 2 diabetes, as well as people at risk for diabetes before onset of symptoms, ${ }^{1,3,4)}$ Multiple variables may modulate a patient's diabetes control and risk for complications. The purpose of this pilot study was to explore how the PI:C ratio in children with recent-onset type 1 diabetes (within 5 years of diagnosis) compared to those with no diabetes and to investigate the effect of age on PI:C ratio.

\section{Materials and methods}

\section{Participants and research design}

This single-visit cross-sectional study was approved by the Pennington Biomedical Research Center Institutional Review Board (Study \#2016-056). Participants were asked to report to the Pennington Biomedical Outpatient Clinic in the morning after 10 hours fasting. Parental consent and participant assent were obtained prior to any study procedures. Children/ adolescents from 10-19 years of age who had been diagnosed with type 1 diabetes within the past 5 years (based on both insulin dependence and self-report) were included. In addition, children and adolescents of similar ages with no diabetes were also recruited as a control group. In the type 1 diabetes group, the major exclusion criteria were pregnancy, use of nicotine products, and use of any medications other than insulin that could alter glucose metabolism.

Address for correspondence:

Daniel S. Hsia, MD

Cennington Biomedical Research

Center, 6400 Perkins Rd., Baton

Rouge, LA 70808, USA

Tel: $+1-225-763-2831$

Fax: +1-225-763-3022

E-mail: daniel.hsia@pbrc.edu

https://orcid.org/0000-0003-28323429 
In addition to blood analysis, measurements of body weight and height were recorded to calculate a body mass index (BMI) and BMI and height $z$-scores based on current Centers for Disease Control and Prevention data. A medical history questionnaire, including information on duration of diabetes (if applicable) and family history of diabetes, was administered. Glucose, C-peptide, proinsulin, and hemoglobin Alc (HbAlc) levels were measured and reported by the Pennington Biomedical Clinical Research Laboratory.

Nine children/adolescents diagnosed with type 1 diabetes and 10 children/adolescents without diabetes were recruited for the study. HbAlc and glucose (Beckman Coulter, Brea, CA, USA) were measured to assess glucose control. Proinsulin was assessed using enzyme-linked immunosorbent assay (Mercodia, Uppsala, Sweden), while C-peptide level was measured by immunoassay with chemiluminescent detection (Siemens, Malvern, PA, USA). The proinsulin assay showed $<0.03 \%$ cross reactivity with insulin and $<0.006 \%$ with C-peptide; whereas, the C-peptide assay showed $10 \%$ cross reactivity with proinsulin. The lowest limit reportable for proinsulin was 3.53 $\mathrm{pmol} / \mathrm{L}$, while the lowest limit reportable for C-peptide was 0.1 $\mathrm{ng} / \mathrm{mL}$. For values below the lowest limit reportable, we assigned a value of one-half the lower limit of detection. ${ }^{3)}$ Molar ratios of proinsulin and C-peptide were calculated and multiplied by 100 to obtain PI:C ratio. Participants were compensated for their participation

\section{Statistical analyses}

Statistical analyses were completed using SAS/STAT software, ver. 9.4, of the SAS System for Windows (Cary, NC, USA). All tests were performed with significance level $\alpha=0.05$, and the findings were considered significant when $P<\alpha$. Due to the small sample size and nonnormality of measured variables, the distributions of age, BMI $z$-score, height $\mathrm{z}$-score, HbAlc, fasting glucose, proinsulin, $\mathrm{C}$-peptide, and PI:C ratio were compared between groups via Wilcoxon rank-sum tests; the results are reported as medians. Chi-square tests were used to compare sex and race. The Pearson correlation between PI:C ratio and age was calculated for the diabetes group and is presented as an $r^{2}$ value.

\section{Results}

A summary of the study population is shown in Table 1. There were no differences in median age, median BMI $z$-score, median height $z$-score, sex, or race between the groups. The median duration of diabetes was 20 months, with a range from 3-47 months. The median HbAlc result in the type 1 diabetes group was $7.3 \%$ (56 mmol/mol), compared to $5.1 \%$ (32 mmol/ $\mathrm{mol})$ in the nondiabetes group $(P<0.01)$, while the median fasting glucose value of the type 1 diabetes group was $209 \mathrm{mg}$ / $\mathrm{dL}$ compared to $88 \mathrm{mg} / \mathrm{dL}$ in the nondiabetes group $(P<0.01)$. The median PI:C ratio was significantly different between the type 1 diabetes and nondiabetes groups (6.24\% vs. $1.46 \%$, $P<0.01)$. In the type 1 diabetes group, there were no statistically significant correlations between duration of type 1 diabetes and PI:C ratio or between $\mathrm{HbAlc}$ and PI:C ratio. Further characterization of the type 1 diabetes group is presented in Table 2 . In the type 1 diabetes group, a negative correlation was observed between PI:C ratio and age, as shown in Fig. $1\left(r^{2}=0.45\right.$,

Table 1. Study population

\begin{tabular}{|c|c|c|c|}
\hline Variable & Type 1 diabetes & No diabetes & $P$-value \\
\hline Age (yr) & $12(10-19)$ & $13.5(10-16)$ & 0.43 \\
\hline BMI z-score & 0.52 (-0.16 to 2.35$)$ & $0.85(-0.32$ to 2.57$)$ & 0.78 \\
\hline Height z-score & 0.55 (-0.16 to 2.11$)$ & $-0.08(-1.86$ to 3.19$)$ & 0.21 \\
\hline Sex & & & 0.76 \\
\hline Female & $3(33.3)$ & $4(40.0)$ & \\
\hline Male & $6(66.7)$ & $6(60.0)$ & \\
\hline Race & & & 0.84 \\
\hline Black & $4(44.4)$ & $4(40.0)$ & \\
\hline White & $5(55.6)$ & $6(60.0)$ & \\
\hline $\begin{array}{l}\text { Diabetes duration } \\
\text { (mo) }\end{array}$ & $20(3-47)$ & - & \\
\hline $\mathrm{HbA1c}(\%)$ & $7.3(6.2-14.4)$ & $5.1(4.7-5.4)$ & $<0.01$ \\
\hline $\begin{array}{l}\text { Fasting glucose } \\
(\mathrm{mg} / \mathrm{dL})\end{array}$ & $209(108-545)$ & $88(80-96)$ & $<0.01$ \\
\hline Proinsulin (pmol/L) & $7.90(1.76-36.36)$ & $10.79(4.65-19.62)$ & 0.27 \\
\hline C-peptide (ng/mL) & $0.40(0.05-1.3)$ & $2.25(1.1-4.1)$ & $<0.01$ \\
\hline $\mathrm{PI}: C$ ratio (\%) & $6.24(2.99-10.35)$ & $1.46(0.54-1.91)$ & $<0.01$ \\
\hline
\end{tabular}

Values are presented as median (range) or number (\%).

$\mathrm{BMI}$, body mass index; $\mathrm{HbA} 1 \mathrm{C}$, hemoglobin $\mathrm{A} 1 \mathrm{C}$; $\mathrm{Pl}$ :C, proinsulin to C-peptide.

Table 2. Characterization of type 1 diabetes participants

\begin{tabular}{|c|c|c|c|c|c|c|c|}
\hline Age (yr) & Diabetes duration (mo) & Sex & Race & $\mathrm{HbA1c}(\%)$ & Proinsulin (pmol/L) & C-peptide $(\mathrm{ng} / \mathrm{mL})$ & PI:C ratio (\%) \\
\hline 11 & 3 & $F$ & W & 6.2 & 14.55 & 0.7 & 6.24 \\
\hline 10 & 39 & M & W & 7.8 & 1.76 & 0.05 & 10.35 \\
\hline 13 & 45 & $\mathrm{~F}$ & $\mathrm{AA}$ & 14.4 & 1.76 & 0.1 & 5.33 \\
\hline 16 & 4 & M & W & 6.7 & 7.90 & 0.7 & 3.39 \\
\hline 10 & 4 & $\mathrm{~F}$ & W & 7.3 & 36.36 & 1.3 & 8.40 \\
\hline 10 & 43 & M & $\mathrm{AA}$ & 6.3 & 1.76 & 0.05 & 10.35 \\
\hline 13 & 18 & M & $\mathrm{AA}$ & 6.8 & 11.95 & 1.2 & 2.99 \\
\hline 12 & 20 & M & $\mathrm{AA}$ & 9.6 & 8.79 & 0.4 & 6.61 \\
\hline 19 & 47 & M & W & 10.0 & 1.76 & 0.1 & 5.33 \\
\hline
\end{tabular}

HbA1c, hemoglobin A1c; W, White; AA, African American; PI:C, proinsulin to C-peptide. 


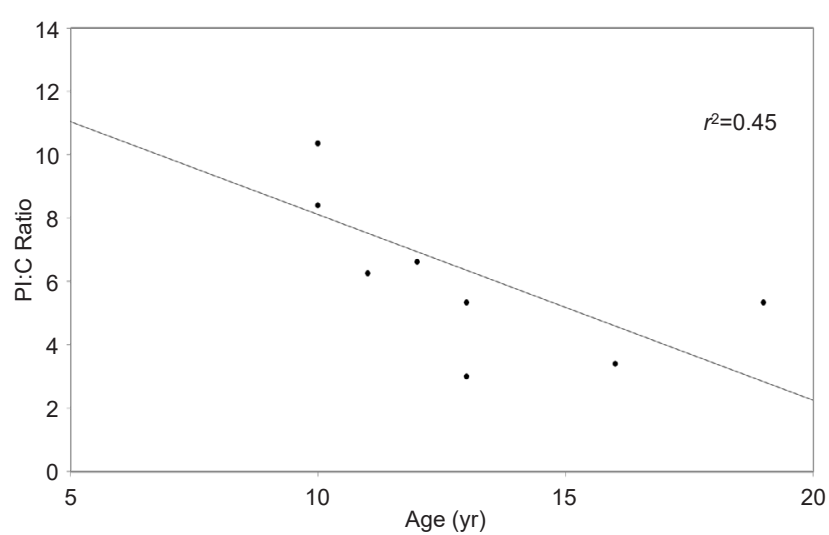

Fig. 1. A significant negative correlation was observed between PI:C ratio and age. PI:C, proinsulin to C-peptide.

$P=0.05)$, and this correlation was even more pronounced when adjusted for duration of diabetes $\left(r^{2}=0.61, P=0.02\right)$.

\section{Discussion}

While we recognize the small number of participants in our study, our results support previous findings that PI:C ratio is clearly elevated in patients diagnosed with type 1 diabetes. ${ }^{5}$ Our results also reinforce previous reports that children with type 1 diabetes who are diagnosed at a younger age have comparatively greater $\beta$-cell dysfunction (as indicated by higher PI:C ratio). ${ }^{6}$ The participants in our study ranged in age from 10-19 years, and they had been diagnosed with type 1 diabetes within the past 5 years (range, 3-47 months). However, we were unable to determine which participants were still in the "honeymoon phase" (when the remaining islet cells start producing insulin temporarily once insulin therapy is initiated), which may have impacted our results. Younger age correlating with higher PI:C ratio is consistent with more aggressive type 1 diabetes at a younger age. ${ }^{7)}$ One hypothesis to explain this trend could be that there are fewer islet cells and a greater degree of insulitis with a higher proportion of $\mathrm{CD} 20+\mathrm{B}$ cells present within the inflamed islets of younger children compared to older adolescents with type 1 diabetes.

Type 1 diabetes is characterized by immune-mediated destruction of insulin-producing $\beta$-cells in the pancreas and leads to lifelong insulin dependence. Thus, it is important to identify biomarkers that reflect $\beta$-cell stress and/or death to improve our ability to optimally time immunomodulatory and other interventions for type 1 diabetes. ${ }^{8)}$ The PI:C ratio may be an important biomarker because accumulation and secretion of inadequately processed proinsulin molecules are hallmarks of $\beta$-cell dysfunction. While antibody testing remains a major determinant of type 1 diabetes diagnosis, PI:C ratio may help to identify high-risk individuals currently experiencing pre-symptomatic $\beta$-cell death. ${ }^{9}{ }^{9}$ Sims et al. ${ }^{3)}$ compared PI:C level between antibody-positive individuals who progressed to diabetes and those who did not. The median PI:C ratio of progressors $\leq 10$ years old $(2.4 \%)$ was triple that of nonprogressors $(0.8 \%)$, while the older progressors exhibited less drastically elevated PI:C ratio. ${ }^{3)}$ These observations demonstrate the effect of age on aggressiveness of type 1 diabetes progression. The higher PI:C ratios in our study are likely a reflection of a greater degree of $\beta$-cell stress in patients with established type 1 diabetes compared to those at risk for developing type 1 diabetes.

While PI:C ratio has been shown to be a good predictor for progression to type 1 diabetes, less is known about its utility in patients already diagnosed with type 1 diabetes and in other diabetes populations. Future research should consider a longitudinal assessment of PI:C ratio and other markers of $\beta$-cell stress at multiple time points in a large number of patients with type 1 or type 2 diabetes who have been diagnosed at various ages. A better understanding of biomarkers of $\beta$-cell dysfunction, such as PI:C ratio, may go a long way toward further understanding progression of types 1 and 2 diabetes. Comparisons could be made among age groups to determine variations in $\beta$-cell stress and their effects on insulin therapy.

In this small cohort, a higher degree of $\beta$-cell dysfunction indicated by a higher PI:C ratio was seen in younger children with type 1 diabetes. This trend may indicate that younger children are more prone to $\beta$-cell dysfunction compared to older children and adolescents. Future longitudinal studies of PI:C ratio in a variety of patient populations may help identify its potential as an indicator of prolonged honeymoon at diagnosis, as a biomarker of $\beta$-cell dysfunction in diabetes treatment trials, and as a predictor of risk for long-term diabetes complications.

\section{Ethical statement}

This single-visit cross-sectional study was approved by the Pennington Biomedical Research Center Institutional Review Board (Study \#2016-056). Parental consent and participant assent were obtained prior to any study procedures.

\section{Conflict of interest}

No potential conflict of interest relevant to this article was reported.

\section{Acknowledgements}

This work was funded by the Pennington Biomedical Research Foundation and was supported in part by U54 GM104940 from the National Institute of General Medical Sciences of the National Institutes of Health, which funds the Louisiana Clinical and Translational Science Center. The content is solely the responsibility of the authors and does not necessarily represent the official views of the National Institutes of Health. Data from this manuscript were submitted to the 
77th Scientific Sessions of the American Diabetes Association and were accepted for publication only.

\section{References}

1. Schölin A, Nyström L, Arnqvist H, Bolinder J, Björk E, Berne C, et al. Proinsulin/C-peptide ratio, glucagon and remission in new-onset Type 1 diabetes mellitus in young adults. Diabet Med 2011;28:156-61.

2. Evans-Molina C, Hatanaka M, Mirmira RG. Lost in translation: endoplasmic reticulum stress and the decline of $\beta$-cell health in diabetes mellitus. Diabetes Obes Metab 2013;15 Suppl 3:159-69.

3. Sims EK, Chaudhry Z, Watkins R, Syed F, Blum J, Ouyang $\mathrm{F}$, et al. Elevations in the fasting serum proinsulin-to-Cpeptide ratio precede the onset of type 1 diabetes. Diabetes Care 2016;39:1519-26.

4. Loopstra-Masters RC, Haffner SM, Lorenzo C, Wagenknecht LE, Hanley AJ. Proinsulin-to-C-peptide ratio versus proinsulin-to-insulin ratio in the prediction of incident diabetes: the Insulin Resistance Atherosclerosis Study (IRAS). Diabetologia 2011;54:3047-54.

5. Ludvigsson J, Heding L. Abnormal proinsulin/C-peptide ratio in juvenile diabetes. Acta Diabetol Lat 1982;19:351-8.

6. Watkins RA, Evans-Molina C, Terrell JK, Day KH, Guindon L, Restrepo IA, et al. Proinsulin and heat shock protein 90 as biomarkers of beta-cell stress in the early period after onset of type 1 diabetes. Transl Res 2016;168:96-106.

7. Leete P, Willcox A, Krogvold L, Dahl-Jørgensen K, Foulis AK, Richardson SJ, et al. Differential insulitic profiles determine the extent of $\beta$-cell destruction and the age at onset of type 1 diabetes. Diabetes 2016;65:1362-9.

8. Mirmira RG, Sims EK, Syed F, Evans-Molina C. Biomarkers of $\beta$-cell stress and death in type 1 diabetes. Curr Diab Rep 2016;16:95.

9. Truyen I, De Pauw P, Jørgensen PN, Van Schravendijk C, Ubani O, Decochez K, et al. Proinsulin levels and the proinsulin:c-peptide ratio complement autoantibody measurement for predicting type 1 diabetes. Diabetologia 2005;48:2322-9. 